\title{
Abemaciclib Does Not Have a Clinically Meaningful Effect on Pharmacokinetics of CYP1A2, CYP2C9, CYP2D6, and CYP3A4 Substrates in Patients with Cancer $^{\circledR}$
}

\author{
(1)P. Kellie Turner, Stephen D. Hall, Sonya C. Chapman, Jessica L. Rehmel, Jane E. Royalty, \\ Yingying Guo, and Palaniappan Kulanthaivel
}

\author{
Eli Lilly and Company, Indianapolis, Indiana (P.K.T., S.D.H., S.C.C., J.L.R., Y.G., P.K.) and Covance Early Clinical Development, \\ Madison, Wisconsin (J.E.R.)
}

Received January 10, 2020; accepted May 19, 2020

\section{ABSTRACT}

Abemaciclib is an orally administered, potent inhibitor of cyclindependent kinases 4 and 6 and is metabolized extensively by CYP3A4. The effects of abemaciclib on several CYPs were qualified in vitro and subsequently evaluated in a clinical study. In vitro, human hepatocytes were treated with vehicle, abemaciclib, or abemaciclib metabolites [N-desethylabemaciclib (M2) or hydroxyabemaciclib (M20)]. mRNA levels for eight CYPs were measured using reversetranscription quantitative polymerase chain reaction, and, additionally, catalytic activities for three CYPs were determined. In the clinical study, adult patients with cancer received a drug cocktail containing CYP substrates [midazolam (3A), warfarin (2C9), dextromethorphan (2D6), and caffeine (1A2)] either alone or in combination with abemaciclib. Plasma pharmacokinetics (PK) samples were analyzed for all substrates, caffeine metabolite paraxanthine, and abemaciclib; polymorphisms of CYP2C9, CYP2D6, CYP3A4, and CYP3A5 were evaluated. In vitro, downregulation of CYP mRNA, including 1A2, 2B6, 2C8, 2C9, 2D6, and 3A, by abemaciclib and/or M2 and M20 was observed at clinically relevant concentrations. In humans, abemaciclib did not affect the PK of CYP2D6 or CYP2C9 substrates. Minor statistically significant but clinically irrelevant changes were observed for midazolam [area under the concentration versus time curve from zero to infinity $\left(A \cup C_{0-i n f}\right)(13 \%$ lower), $C_{\max }\left(15 \%\right.$ lower)], caffeine [AUC ${ }_{0-\text { inf }}(56 \%$ higher)], and paraxanthine: caffeine [area under the concentration versus time curve from 0 to 24 hours ratio (was approximately $30 \%$ lower)]. However, given the magnitude of the effect, these changes are not considered clinically relevant. In conclusion, the downregulation of CYP mRNA mediated by abemaciclib in vitro did not translate into clinically meaningful drug-drug interactions in patients with cancer.

\section{SIGNIFICANCE STATEMENT}

Despite observations that abemaciclib alters the mRNA of various CYP isoforms in vitro, a clinical study using a drug cocktail approach found no clinically meaningful drug-drug interactions between abemaciclib and a range of CYP substrates [midazolam (CYP3A4), S-warfarin (CYP2C9), dextromethorphan (CYP2D6), and caffeine (CYP1A2)]. This lack of translation suggests greater understanding of mechanisms of CYP downregulation is needed to accurately predict clinical drug-drug interaction risk from in vitro data.

\section{Introduction}

Abemaciclib is an orally administered, selective cyclin-dependent kinase 4 and cyclin-dependent kinase 6 inhibitor approved for the treatment of hormone receptor-positive, human epidermal growth factor receptor 2-negative advanced or metastatic breast cancer (Dickler et al., 2017). Abemaciclib is primarily metabolized by CYP3A4, producing

The study was sponsored by Eli Lilly and Company.

Disclosures P.K.T., S.D.H., S.C.C., J.Re., and Y.G. are employees and minor stockholders of Eli Lilly and Company. At the time of the research, P.K. was an employee and minor stockholder of Eli Lilly and Company. J.Ro. is an employee at Covance.

https://doi.org/10.1124/dmd.119.090092.

$\$$ This article has supplemental material available at dmd.aspetjournals.org. two major ( $>10 \%$ circulating exposure) equipotent metabolites: $\mathrm{N}$-desethylabemaciclib [LSN2839567 (M2)] and hydroxyabemaciclib [LSN3106726 (M20)].

Given that abemaciclib is dependent on CYP3A4 for elimination, two clinical drug-drug interaction (DDI) studies were performed to assess the impact of CYP3A induction and inhibition on the exposure of abemaciclib and its active metabolites. Rifampin, a strong CYP3A inducer, decreased the area under the concentration versus time curve (AUC) of abemaciclib by 95\% (Kulanthaivel et al., 2016). After dosing with clarithromycin, a strong CYP3A inhibitor, the AUC ratio for abemaciclib was 3.4 (Kulanthaivel et al., 2016; Posada et al., 2017). Accordingly, labeling includes recommendations around the concomitant use of CYP3A inducers and inhibitors (http://uspl.lilly.com/verzenio/verzenio.html\#pi;

ABBREVIATIONS: $\mathrm{AUC}_{0-24}$, area under the concentration versus time curve from 0 to 24 hours; $\mathrm{AUC}_{0-\text { inf }}$, area under the concentration versus time curve from zero to infinity; AUCR, area under the concentration versus time curve ratio; Cl, confidence interval; DDI, drug-drug interaction; ECOG, Eastern Cooperative Oncology Group; $E_{\max }$, maximum extent of induction or downregulation; INR, international normalized ratio; INR AUC ${ }_{(0-96)}$, area under the INR versus time curve from time 0 to 96 hours postdose; LS, least squares; M2, $N$-desethylabemaciclib; M20, hydroxyabemaciclib; MMAE, monomethyl auristatin E; P:C, paraxanthine to caffeine ratio; PCR, polymerase chain reaction; PK, pharmacokinetics; $\mathrm{Q} 12 \mathrm{H}$, every 12 hours; TEAE, treatment-emergent adverse event; $t_{\max }$, time of maximum observed drug concentration. 
https://www.ema.europa.eu/en/documents/scientific-guideline/guidelineinvestigation-drug-interactions_en.pdf). Although the effect of other drugs on abemaciclib is well-characterized, the effects of abemaciclib on the pharmacokinetics of CYP substrate drugs have not been extensively explored.

The incubation of drug and major metabolites with cultured human hepatocytes provides a convenient and efficient approach to assess whether significant CYP induction or downregulation of CYP expression occurs in vitro. The mechanisms responsible for CYP induction have been described in detail (Sun et al., 2017), and the induction response of CYP3A4 mRNA in hepatocytes translates into the clinic (Fahmi and Ripp, 2010). In contrast, although CYP downregulation in vitro has been described (Hariparsad et al., 2017), the generalizability of the mechanisms is unknown, and clinical translation has not been verified. Thus, the detection of CYP downregulation in vitro is generally followed by a clinical drug interaction study requiring multiple doses of the putative inhibitor. This is to allow the target enzymes to approach a new steady state, which is estimated to be 7 days based on CYP degradation half-lives of 24-36 hours (Yang et al., 2008).

In the present study, we evaluated the effects of abemaciclib and its major active metabolites, M2 and M20, on CYP1A2, CYP2B6, CYP2C8, CYP2C9, CYP2C19, CYP2D6, and CYP3A4 and CYP3A5 mRNA expression in cultured human hepatocytes. Based on the in vitro results, we conducted a clinical study in patients with cancer to assess the effects of multiple doses of abemaciclib on the pharmacokinetics (PK) of a cocktail of CYP substrates, namely midazolam (CYP3A4), S-warfarin (CYP2C9), dextromethorphan (CYP2D6), and caffeine (CYP1A2).

\section{Materials and Methods}

\section{In Vitro Studies}

Abemaciclib, M2, and M20 were supplied by Eli Lilly and Company (Indianapolis, IN). Cryopreserved primary human hepatocytes and supplemented modified Eagle's medium (with Dr. Chee's modification; MCM+) were obtained from XenoTech LLC (Lenexa, KS), with lot-specific details given in Supplemental Table 1. Phenacetin, acetaminophen, bupropion hydrochloride, midazolam, omeprazole, phenobarbital, and rifampicin were obtained from Sigma Aldrich (St. Louis, MO). Hydroxybupropion and 1'-hydroxymidazolam were from Cerilliant (Round Rock, TX). Stable-label internal standards for acetaminophen, hydroxybupropion, and 1'-hydroxymidazolam were from a proprietary source. Reagents for RNA isolation and PCR were from Applied Biosystems and Qiagen.

Primary human hepatocytes in culture were treated with vehicle, abemaciclib, or M2 or M20 for 48 hours. Abemaciclib was incubated with a single lot of cells, whereas M2 and M20 were each incubated with three lots of cells. Quantitative evaluation of cytotoxicity was measured using a lactate dehydrogenase release assay (Roche Diagnostics, Indianapolis, IN). mRNA levels for CYP1A2, CYP2B6, CYP2C8, CYP2C9, CYP2C19, CYP2D6, CYP3A4, and CYP3A5 as well as the endogenous control, glyceraldehyde 3-phosphate dehydrogenase, were measured using reverse-transcription quantitative PCR with specific assays listed in Supplemental Table 2. Catalytic activities for CYP1A2, CYP2B6, and CYP3A4 were measured using selective catalytic assays for CYP1A2 (phenacetin $O$-deethylation to acetaminophen), CYP2B6 (bupropion hydroxylation), and CYP3A4 (midazolam 1'-hydroxylation) as described in Supplemental Table 3. Omeprazole $(50 \mu \mathrm{M})$ served as the positive control for aryl hydrocarbon receptor-mediated induction of CYP1A2, phenobarbital $(750 \mu \mathrm{M})$ was the positive control for constitutively active receptor-mediated induction of CYP2B6, and rifampicin $(20 \mu \mathrm{M})$ was the positive control for pregnane $\mathrm{X}$ receptor-mediated induction of the remaining inducible CYPs. Data are reported for noncytotoxic concentrations as determined by lactate dehydrogenase release, increases in cycle threshold values for glyceraldehyde 3-phosphate dehydrogenase, and/or morphologic observations. Criteria for induction were concentrationdependent increases in mRNA expression of greater than or equal to 2-fold and a response greater than or equal to $20 \%$ of the positive control response. Concentration-dependent decreases in mRNA of greater than or equal to $50 \%$ were considered downregulation.
To model the effect of downregulation of CYP3A4 on the oral clearance of midazolam, nonlinear regression using a four-parameter logistic model was used to estimate the maximum extent of induction or downregulation $\left(\mathrm{E}_{\max }\right)$ and $\mathrm{EC}_{50}$ (version 7; Graphpad Prism, San Diego, CA). The $\mathrm{E}_{\max }$ and $\mathrm{EC}_{50}$ for CYP3A4 were entered in a simplified mechanistic static net effect model (eq. 1) (Fahmi et al., 2008) to predict the AUCR (ratio of AUC with and without downregulation) for orally administered midazolam.

$$
A U C R=\frac{1}{\left(1-\frac{E_{\max } \times[]_{g}}{[I]_{g}+E C_{50}}\right) \times\left(1-F_{g}\right)+F_{g}} \times \frac{1}{\left(1-\frac{E_{\max } \times[]_{h}}{\left[I_{h}+E C_{50}\right.}\right) \times f_{m}+1-f_{m}}
$$

in which $\mathrm{f}_{\mathrm{m}}$ is the fraction of systemic clearance of midazolam via CYP3A4 (set at $0.9), \mathrm{F}_{\mathrm{g}}$ is the fraction of midazolam escaping metabolism in the gut (set at 0.5 ), $[\mathrm{I}]_{\mathrm{H}}$ is the concentration of perpetrator drug at the liver (eq. 2), and $[\mathrm{I}]_{\mathrm{G}}$ is the concentration of perpetrator drug at the intestine (eq. 3), with inputs as shown in Supplemental Table 4 (Ito et al., 1998; Rostami-Hodjegan and Tucker, 2004, United States Food and Drug Administration, 2017; Tate et al., 2018).

$$
\begin{aligned}
& {[]_{\mathrm{h}}=\mathrm{f}_{\mathrm{u}, \mathrm{p}} \times\left(\mathrm{C}_{\max }+\mathrm{F}_{\mathrm{a}} \times \mathrm{k}_{\mathrm{a}} \times \text { Dose } / \mathrm{Q}_{\mathrm{h}} / \mathrm{R}_{\mathrm{b}}\right)} \\
& {[\mathrm{I}]_{\mathrm{g}}=\mathrm{F}_{\mathrm{a}} \times \mathrm{k}_{\mathrm{a}} \times \text { Dose } / \mathrm{Q}_{\mathrm{en}}}
\end{aligned}
$$

\section{Clinical Study Design}

This Phase 1, multicenter, open-label, fixed-sequence study (NCT02688088) was conducted in patients with advanced and/or metastatic cancer. The enrolled patients were at least 18 years of age with an Eastern Cooperative Oncology Group (ECOG) score between 0 and 2 and adequate organ function. The study excluded patients who had surgery performed that could affect the absorption or experience emesis that may affect drug PK.

The study was conducted in accordance with ethical principles derived from international ethics guidelines, including the Declaration of Helsinki, the Council for International Organizations of Medical Sciences International Ethical Guidelines, the International Conference on Harmonisation Good Clinical Practice guideline, and applicable laws and regulations.

Treatment. A drug cocktail containing four sensitive CYP substrates was selected based on a subset of the validated Cooperstown $5+1$ cocktail, namely midazolam (CYP3A4), S-warfarin (CYP2C9), dextromethorphan (CYP2D6), and caffeine (CYP1A2) (Chainuvati et al., 2003). A CYP2B6 substrate was not included because of the low number of drugs dependent on CYP2B6 clearance. The cocktail containing $0.2 \mathrm{mg}$ midazolam, $10 \mathrm{mg}$ warfarin, $30 \mathrm{mg}$ dextromethorphan, and $100 \mathrm{mg}$ caffeine was administered orally as a single dose on two occasions: alone on day 1 in period 1 , and in combination with abemaciclib on day 8 of period 2. Abemaciclib was administered continuously at $200 \mathrm{mg} \mathrm{Q12H}$ for 7 days prior to drug cocktail administration in order for abemaciclib steady-state concentrations to be reached (Patnaik et al., 2016) and for any time-dependent changes in CYP activity to take full effect [ 3-5 CYP half-lives (36 hours)] (Fig. 1. Study Design). Abemaciclib dose interruptions or modifications were avoided when possible but were permitted if needed because of individual intolerability. Food consumption was not permitted 1 hour before or after taking abemaciclib or the drug cocktail. Known inducers and/or inhibitors of CYP1A2, CYP2C9, CYP2D6, and CYP3A4 were excluded 14 days prior to drug cocktail administration and throughout periods 1 and 2; participants were also asked to refrain from consuming grapefruit juice, Seville oranges, and St. John's Wort during the same time frame. Dietary caffeine consumption was not permitted 5 days prior to drug cocktail administration and throughout periods 1 and 2. In patients reporting treatment-emergent adverse events (TEAEs), the abemaciclib dose was lowered from $200 \mathrm{mg}$ twice daily to $150 \mathrm{mg}$ twice daily. If the patient had an international normalized ratio (INR) of more than 2 after the first dose of drug cocktail, abemaciclib was only dosed thereafter once the INR had returned to acceptable levels. If patients had an INR greater than 2 in period 1, they may have received the drug cocktail without warfarin in period 2.

\section{Evaluation Methods}

Assays. Human plasma samples were analyzed for abemaciclib, M2, M20, and midazolam (Q2 Solutions, Ithaca, NY) as well as $S$-warfarin, dextromethorphan, 


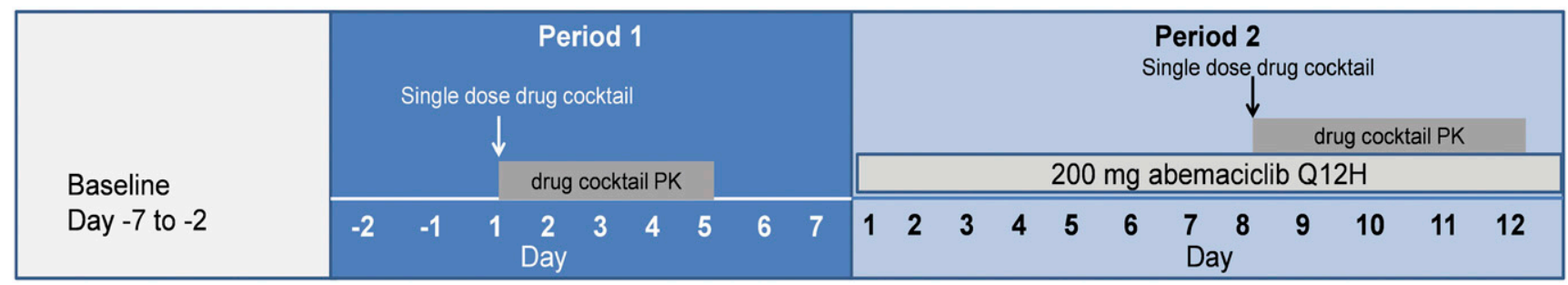

Fig. 1. Study design

caffeine, and paraxanthine (Covance Bioanalytical Services, LLC, Indianapolis, IN) using validated liquid chromatography with tandem mass spectrometric methods.

Pharmacokinetics. PK samples were collected on period 1 day 1 and period 2 day 8 predose and $0.5,1,2,3,4,6,8,12$, and 24 hours postdose for caffeine, paraxanthine, and midazolam, with an additional sample at 48 hours for midazolam. PK samples for dextromethorphan and $S$-warfarin were collected at predose and 1, 2, 4, 6, 8, 10, 24, 48, and 72 hours postdose on day 1 (period 1) and day 8 (period 2), with an additional sample at 96 hours for $S$-warfarin. Predose PK samples were taken in period 2 on days 7-9 for abemaciclib.

Pharmacodynamics. Blood samples were collected predose and 8, 24, 48, 72, and 96 hours postdose on period 1 day 1 and period 2 day 8 to assess INR $\mathrm{AUC}_{0-96}$ and $\mathrm{INR}_{\max }$.

Pharmacogenomics. A blood sample for each patient was collected in vacutainer EDTA tubes either during the baseline visit or predose (period 1 day 1). Genomic DNA was then extracted for CYP2C9, CYP2D6, CYP3A4, and CYP3A5, and genotyping was performed by validated TaqMan quantitative PCR assays (Thermo Fisher Scientific, Waltham, MA) and long-range PCR assays (Fijal et al., 2015) at Covance Genomics Laboratory (Redmond, WA). These included alleles of $C Y P 2 D 6 * 2, * 3, * 4, * 5, * 6, * 7, * 8, * 9, * 10, * 17, * 29, * 41$, and duplications; $C Y P 2 C 9 * 2$ and $* 3$; and $C Y P 3 A 4 * 3, * 7, * 20$, and $* 22$ as well as $C Y P 3 A 5 * 3$, *4, $* 5$, $* 6$, and $* 7$. The functional-determining genetic variant for each star allele was selected and assayed according to the Human Cytochrome P450 (CYP) Allele Nomenclature Database. Allele frequencies were tested for Hardy-Weinberg equilibrium within each ethnic group and considered significant at $P<0.0001$. Patients were classified into metabolizer groups for each CYP based on the combination of alleles associated with their genotype (Ingelman-Sundberg et al., 2007; Johnson et al., 2011; Birdwell et al., 2015; Hicks et al., 2017). Patients who were poor metabolizers for any CYP were evaluated for exclusion from the DDI statistical analysis of the respective probe substrate.

\section{Statistical Methods}

Pharmacokinetic and Pharmacodynamic Endpoints. Midazolam, $S$-warfarin, dextromethorphan, caffeine, and paraxanthine PK (e.g., $\mathrm{C}_{\max }, \mathrm{AUC}_{0 \text {-inf }}$, and $\mathrm{t}_{\max }$ ) and $S$-warfarin pharmacodynamics (INR $\mathrm{AUC}_{(0-96)}$ or $\mathrm{INR}_{\max }$ ) were calculated by standard noncompartmental methods (Version 6.4; Phoenix WinNonlin). The ratio between paraxanthine and caffeine exposure was calculated for the 6-hour concentration timepoint and for $\mathrm{AUC}_{0-24}$. Logtransformed $\mathrm{C}_{\max }$ and $\mathrm{AUC}_{0 \text {-inf }}$ estimates were evaluated in a linear mixedeffects ANOVA model with a fixed effect for treatment and a random effect for subject. The treatment differences were back-transformed to present the ratio of geometric least squares means and the corresponding $90 \%$ confidence intervals (CIs). The $t_{\max }$ was analyzed using a Wilcoxon signed rank test. Estimates of the median difference based on the observed medians, $90 \% \mathrm{CIs}$, and $P$ values from the Wilcoxon test were calculated.

Safety. Adverse event terms and severity grades were assigned by the investigator using Common Terminology Criteria for Adverse Events (Version 4.0).

\section{Results}

\section{In Vitro Studies}

First, the effect of abemaciclib and metabolites on catalytic activity of CYP enzymes was assessed. In human liver microsomes, abemaciclib and its major circulating metabolites, M2 and M20, did not inhibit the catalytic activities of CYP1A2, CYP2B6, CYP2C8, CYP2C9, CYP2C19, CYP2D6, or CYP3A4 either directly or in a timedependent manner at clinically relevant circulating concentrations for abemaciclib $(0.59 \mu \mathrm{M}), \mathrm{M} 2(0.27 \mu \mathrm{M})$, and M20 $(0.38 \mu \mathrm{M})$. No reversible or time-dependent inhibition of CYPs was expected in the liver based on the in vitro data from human liver microsomes for abemaciclib, M2, or M20. However, because of poor solubility, concentrations achieved in vitro were not sufficiently high $(12.5 \mu \mathrm{M}$ for abemaciclib) to rule out CYP3A4 inhibition at the intestine (relevant concentration $0.1 *$ dose $/ 250 \mathrm{ml}=160 \mu \mathrm{M})(\mathrm{https}: / / \mathrm{www} . \mathrm{ema}$. europa.eu/en/documents/scientific-guideline/guideline-investigationdrug-interactions_en.pdf).

We next assessed the effect of abemaciclib and metabolites on mRNA expression. Downregulation of CYP mRNA, including 1A2, 2B6, 2C8, 2C9, 2D6, 3A4, and 3A5 (but not 2C19), by abemaciclib and/or M2 and M20 was observed in the in vitro study conducted in human hepatocytes (Fig. 2, A-C). This mRNA downregulation was concentrationdependent, greater than $50 \%$, and not due to cytotoxicity to cells according to evaluation of three endpoints. Furthermore, in studies in human hepatocytes, abemaciclib and metabolites did not induce the catalytic activities of CYP1A2, CYP2B6, or CYP3A4. Instead, activity for all three CYPs decreased; CYP3A4 activity is shown as an example in Fig. 2D (http://uspl.lilly.com/verzenio/verzenio.html\#pi). An overall tabular summary of the interpretation of the data for abemaciclib, M2, and $\mathrm{M} 20$, including selected $\mathrm{E}_{\max }$ and $\mathrm{EC}_{50}$ values, is shown in Supplemental Table 1, and the mean data, presented as $\%$ change from $0.1 \%$ DMSO (vehicle) control, is shown in Supplemental Table 5.

In the case of CYP2D6, both induction (up to 2.87-fold change) and marked decreases (up to $79.3 \%$ ) in mRNA levels were observed when hepatocytes were treated with abemaciclib across the concentration range (Fig. 2; Supplemental Table 1). For CYP3A4 mRNA, $\mathrm{E}_{\max }$ and $\mathrm{EC}_{50}$ based on fitting of the data for abemaciclib were -0.89 -fold and $0.37 \mu \mathrm{M}$, respectively. Application of the mechanistic static model (eq. 1) predicted an AUCR of 2.0 for oral midazolam due to CYP3A4 downregulation by abemaciclib. When M2 and M20 were included in the calculation (affecting midazolam model systemic clearance only), the AUCR increased to 2.1 .

\section{Clinical Trial}

Demographics and Disposition. The majority of the 44 patients enrolled in the study were Caucasian $(91 \%)$ with a mean age of 60 years (range: 37-78 years) (Table 1). Most patients had an ECOG status of $0(27.3 \%)$ or $1(68.2 \%)$.

Of the 44 patients who entered the study and received at least one dose of study drug, 37 completed period 2. There were two patients who discontinued prior to day 1 of period 2, one because of fatal disease progression and one because of a TEAE of increased bilirubin.

Pharmacokinetics. Up to 44 patients were included in the PK analysis for period 1, and up to 37 were included in period 2. The PK 
A

CYP1A2 mRNA

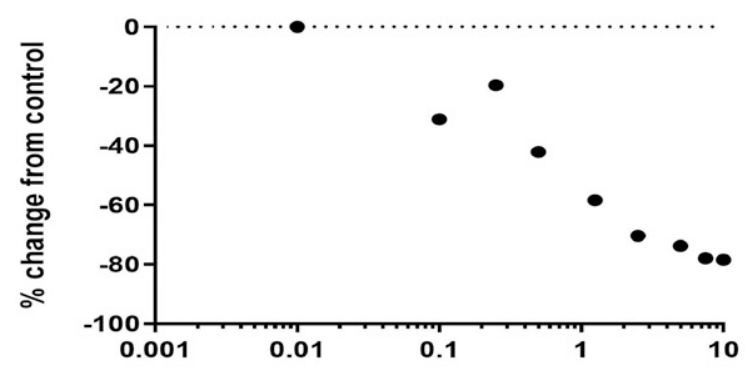

concentration $(\mu \mathrm{M})$

C

CYP3A4 mRNA

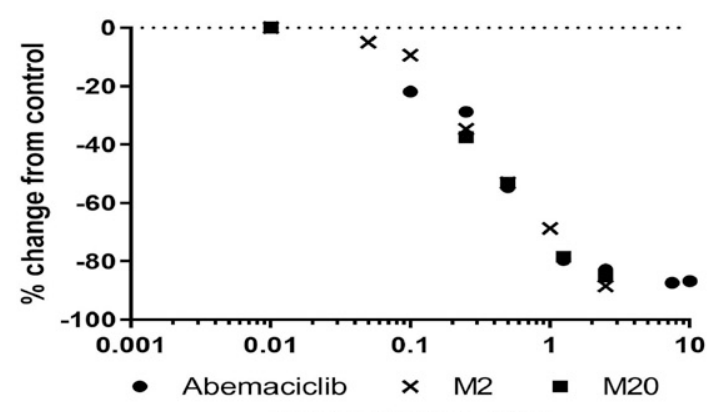

concentration $(\mu \mathrm{M})$
B

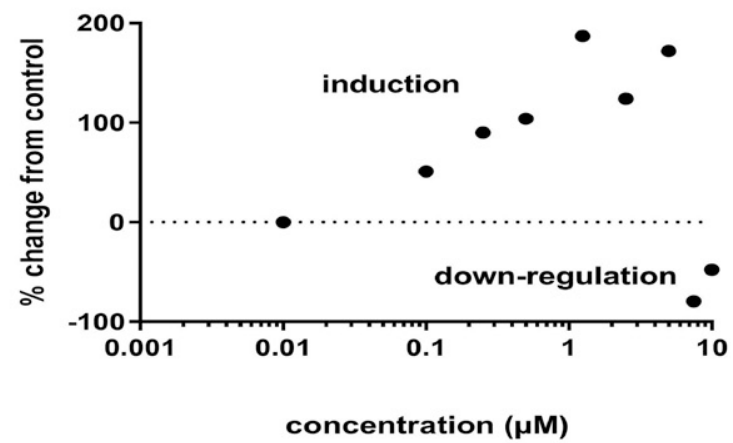

D

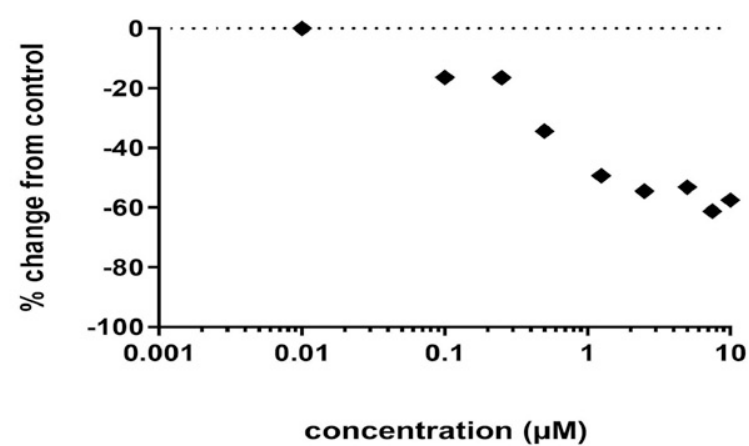

Fig. 2. Representative data showing the effects of abemaciclib and metabolites (M2 and M20) on CYP1A2, CYP2B6, and CYP3A4 mRNA in human hepatocytes incubated for 48 hours. (A) The effects of abemaciclib on CYP1A2 mRNA. (B) The mixed effects of abemaciclib on CYP2D6 mRNA. (C) The effects of abemaciclib (circles), M2 (x), and M20 (squares) on CYP3A4 mRNA in lot HC3-22. (D) The effects of abemaciclib on CYP3A4 activity. An overall tabular summary of the data for abemaciclib, M2, and M20 is shown in Supplemental Table 1, and mean data at individual concentrations are shown in Supplemental Table 5.

profiles for each analyte in the presence and absence of abemaciclib are shown in Fig. 3. Individual changes in $\mathrm{AUC}_{0-24}$ are shown in Fig. 4.

Midazolam. The midazolam $\mathrm{AUC}_{0-\text { inf }}$ was approximately $13 \%$ lower and the $\mathrm{C}_{\max }$ was approximately $15 \%$ lower when midazolam was administered after multiple doses of abemaciclib versus when midazolam was administered alone with no significant differences in median $t_{\max }$. The lower bounds of the $90 \%$ CIs for the ratios for midazolam AUC $_{0-\text { inf }}$ and $\mathrm{C}_{\max }$ were below 0.8 ( 0.775 and 0.760 , respectively) (Fig. 5; Table 2).

TABLE 1

Demographics of enrolled patients

\begin{tabular}{lc}
\hline Parameter & Overall \\
\hline Age, yr, mean (SD) & $60(11)$ \\
Sex, $n(\%)$ & \\
Female & $24(54.5)$ \\
Male & $20(45.5)$ \\
Body mass index, $\mathrm{kg} / \mathrm{m}^{2}$, mean (SD) & $28.5(5.05)$ \\
Ethnicity, $n(\%)$ & \\
Hispanic or Latino & $2(4.5)$ \\
Not Hispanic or Latino & $41(92.2)$ \\
Unknown & $1(2.3)$ \\
Race, $n(\%)$ & \\
Asian & $1(2.3)$ \\
Black or African American & $1(2.3)$ \\
White & $40(90.9)$ \\
Unknown & $2(4.5)$ \\
ECOG status, $\%$ & \\
0 & 27.3 \\
1 & 68.2 \\
2 & 4.5 \\
\hline
\end{tabular}

$S$-Warfarin. No significant differences in the geometric least squares (LS) mean $S$-warfarin $\mathrm{C}_{\max }$, $\mathrm{AUC}_{0 \text {-inf }}$, or $\mathrm{t}_{\max }$ between warfarin administered alone or in combination with abemaciclib were evident (Fig. 5; Table 2). There were also no significant differences in INR $\mathrm{AUC}_{(0-96)}$ or $\mathrm{INR}_{\max }$ when warfarin was administered after multiple doses of abemaciclib [geometric mean (CV\%), 1.27 (34\%), 1.56 (44\%), respectively] compared with when administered alone [120 (23\%) and $1.43(32 \%)$, respectively].

Dextromethorphan. Based on the CYP2D6 genotyping results, $70.5 \%$ of patients were extensive metabolizers, $9.1 \%$ were intermediate metabolizers, $2.3 \%$ were ultra-rapid metabolizers, $2.3 \%$ were poor metabolizers, and in $9.1 \%$ the genotype could not be interpreted. Data from the patient with poor metabolizer status were excluded from the statistical analysis for dextromethorphan; however, those with unknown status were included in the analysis. Abemaciclib had no statistically significant effects on dextromethorphan $\mathrm{C}_{\max }, \mathrm{AUC}_{0 \text {-inf }}$, or $\mathrm{t}_{\max }$ (Fig. 5; Table 2).

Caffeine/Paraxanthine. The $\mathrm{AUC}_{0 \text {-inf }}$ of caffeine was $56.0 \%$ higher when caffeine was administered after multiple abemaciclib doses compared with administration alone, with the upper limit of the $90 \%$ CI for the ratio of geometric LS mean exceeding the 1.25 boundary; however, there were no significant differences in caffeine $\mathrm{C}_{\max }$ or $\mathrm{t}_{\max }$ (Fig. 5; Table 2). The paraxanthine to caffeine ratio (P:C) decreased by $38.9 \%$ at the P:C 6-hour time point and decreased by $31.7 \%$ for P:C $\mathrm{AUC}_{0-24}$ in the presence of abemaciclib (Table 2).

Noncompliance with caffeine restriction was evident in the data, both before and after drug cocktail administration, as demonstrated by higher than expected levels of caffeine in predose samples and in samples taken after the patient had left the clinical research unit (24 and 48 hours postdose) (Supplemental Fig. 1). Quantifiable predose caffeine 

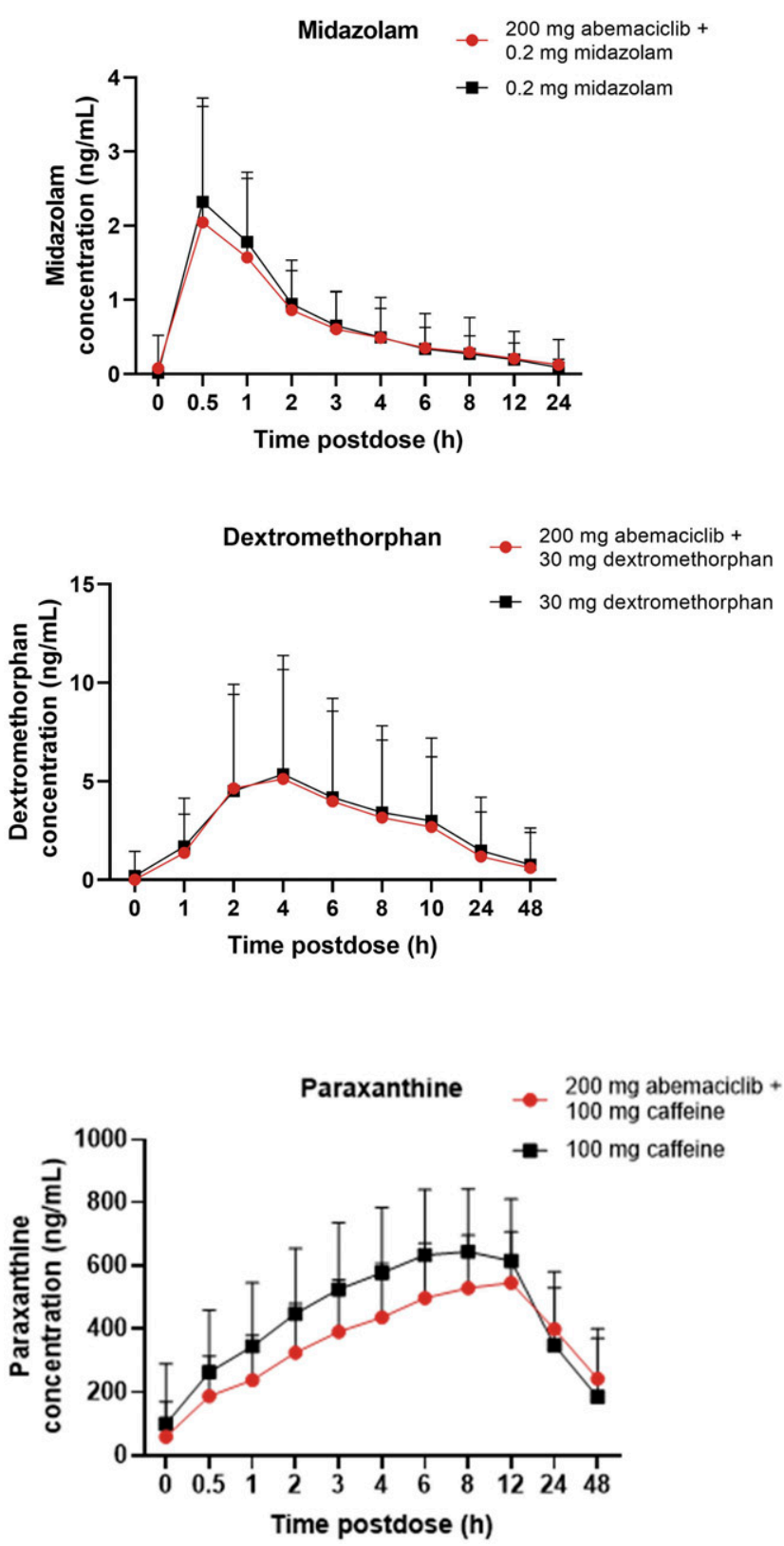
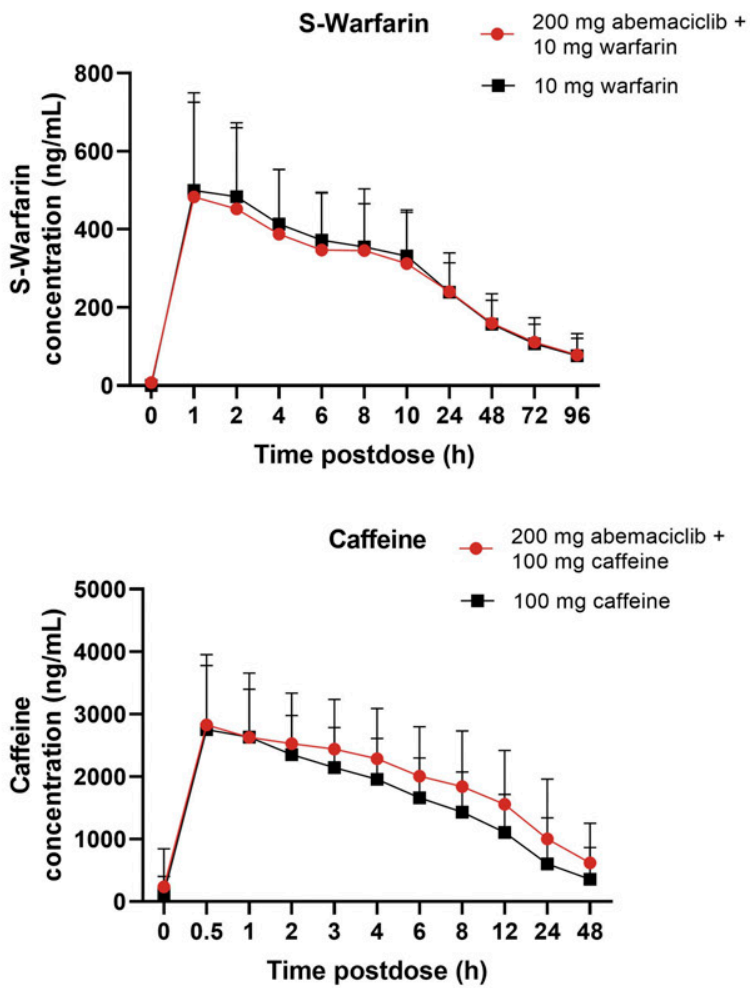

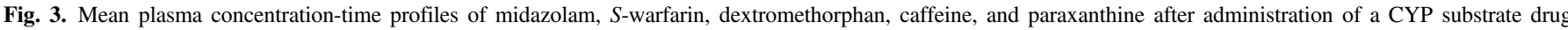

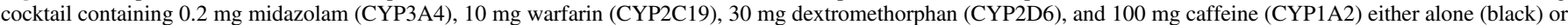
in combination with abemaciclib after 7 days of $200 \mathrm{mg}$ Q12H abemaciclib (red).

concentrations of more than $10 \%$ of $\mathrm{C}_{\max }$ were observed in period $1(n=$ $4)$ and period $2(n=5)$, and two patients exhibited post-clinical research unit increases in caffeine concentrations in both study periods. There was one patient who exhibited both predose caffeine concentrations greater than $10 \%$ of $\mathrm{C}_{\max }$ and rising concentrations after clinical research unit discharge in both study periods.

Abemaciclib. The geometric mean abemaciclib trough concentrations after Q12H abemaciclib oral dosing with $200 \mathrm{mg}$ (the highest approved dose) or $150 \mathrm{mg}$ (if the patient had experienced a dose reduction) were between $453 \mathrm{ng} / \mathrm{ml}(0.89 \mu \mathrm{M})$ and $561 \mathrm{ng} / \mathrm{ml}(1.11 \mu \mathrm{M})$.

Safety. A total of 250 TEAEs were reported, of which the majority $(87.6 \%)$ were Grade 1 or Grade 2 in severity, and $11.2 \%$ of patients experienced greater than or equal to Grade 3 events. Overall, the most common drug-related TEAEs of any grade were diarrhea $(56.8 \%)$, nausea $(25.0 \%)$, and vomiting (25.0\%). Adverse events greater than or equal to
Grade 3 observed included two events of vomiting and five events of diarrhea. Abemaciclib-induced adverse events were generally manageable and monitorable. Four patients reported serious adverse events. This included one patient each with Common Terminology Criteria for Adverse Events Grade 2 abdominal pain, Grade 3 diarrhea $(n=1)$, fractured radius $(n=1)$, and Grade 3 cholangitis $(n=1)$ related to other medical conditions as well as Grade 3 ascites $(n=1)$, which was diseaserelated. The only study drug-related events were abdominal pain and diarrhea. One patient death was reported during period 1 (drug cocktail in the absence of abemaciclib) that was due to disease progression.

\section{Discussion}

Patients receiving abemaciclib for the treatment of cancer are likely to receive other drugs to manage their condition and/or other nonrelated 
Midazolam

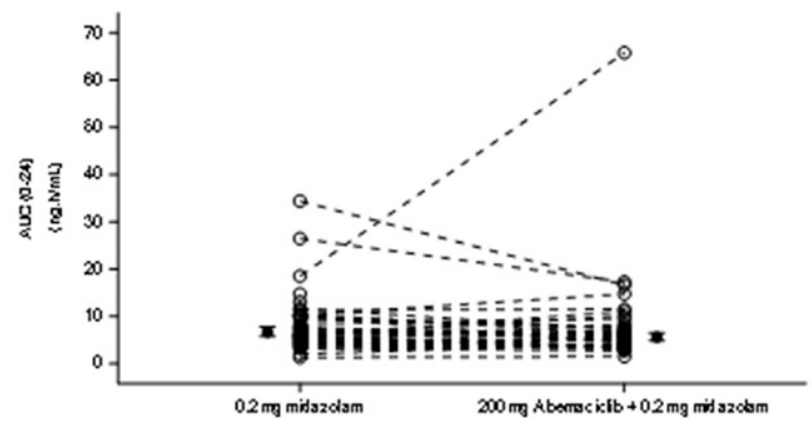

Dextromethorphan

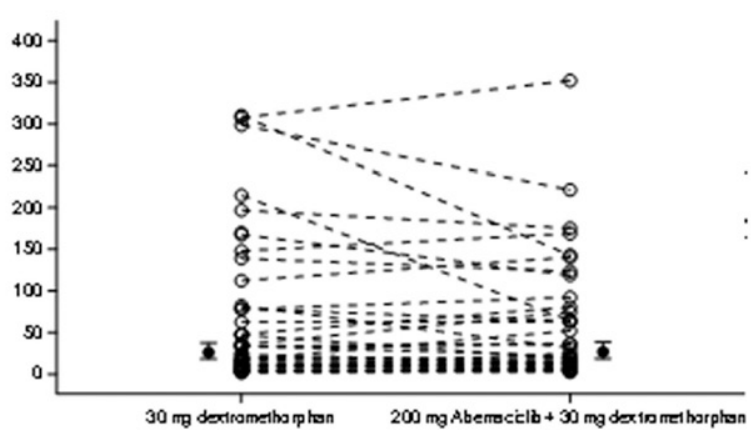

Paraxanthine

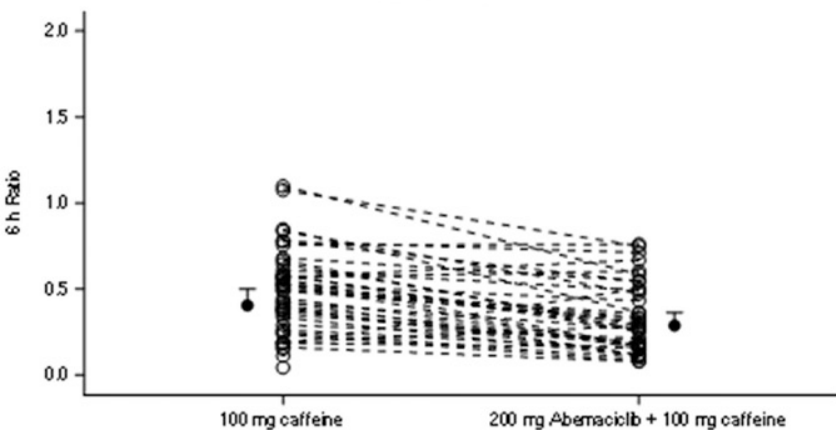

S-Warfarin ogemetrie mes value $(90 \%$ d

individual values

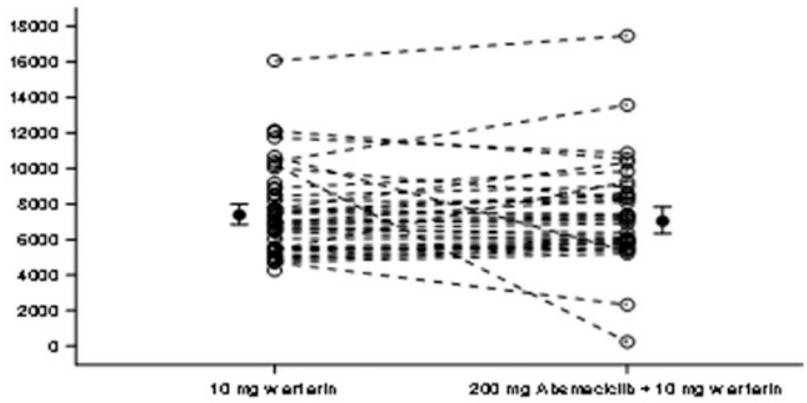

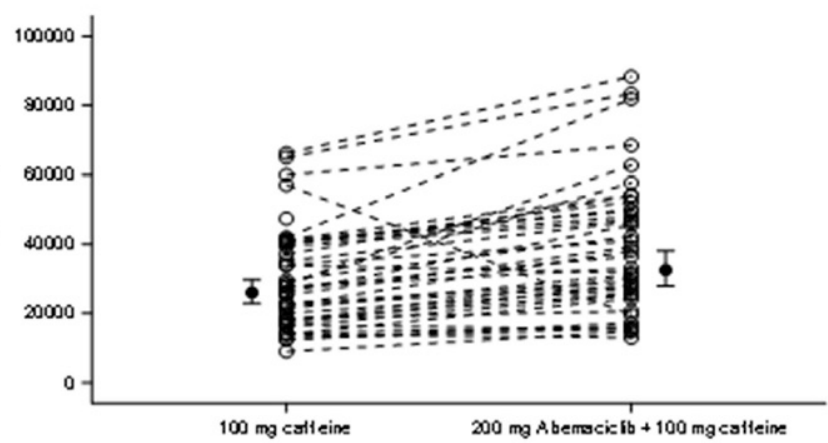

Fig. 4. Individual $\mathrm{AUC}_{0-24}$ of midazolam, $S$-warfarin, dextromethorphan, and caffeine and individual 6-hour paraxanthine:caffeine ratios after administration of a CYP substrate drug cocktail containing $0.2 \mathrm{mg}$ midazolam (CYP3A4), $10 \mathrm{mg}$ warfarin (CYP2C19), $30 \mathrm{mg}$ dextromethorphan (CYP2D6), and 100 mg caffeine (CYP1A2) either alone or in combination with abemaciclib after 7 days of $200 \mathrm{mg}$ Q12H abemaciclib. Individual data are denoted by open circles, individual change is denoted by dotted lines, and the geometric means of the individual data are denoted by filled circles.

illnesses. Many of these concomitant medications are likely to be CYP substrates. The present study investigated the potential in vitro and clinical effect of abemaciclib and its major circulating equipotent metabolites, M2 and M20, on the exposures of other CYP substrate drugs.

During in vitro studies, abemaciclib-mediated concentrationdependent downregulation of several CYPs in cultured human hepatocytes was observed. The mechanisms underlying this observation are unknown, although the similarity of the downregulation pattern across abemaciclib, M2, and M20 suggest a common mechanism may be involved. One possible explanation could be in vitro metabolism of abemaciclib, M2, and M20, which results in a common downregulating species. Poor evidence exists for the translation of in vitro CYP downregulation to in vivo changes in PK. For CYP3A4, some small molecules downregulate mRNA expression in hepatocytes in vitro by binding to and downregulating the nuclear factor pregnane $\mathrm{X}$ receptor, which mediates the upregulation of this enzyme by inducers (Tian, 2013; Burk et al., 2018; Staudinger, 2019). In vivo exploration of the clinical effects of antibody-conjugated momethyl auristatin E (MMAE) (the active moiety of brentuxumab vedotin), another in vitro downregulator of CYP3A4 without reduced cell viability, was hindered by low systemic exposures due to antibody-conjugated MMAE delivery. This lack of effect of MMAE on the clearance of midazolam can be explained by the relevant in vitro concentrations not being achieved in vivo 


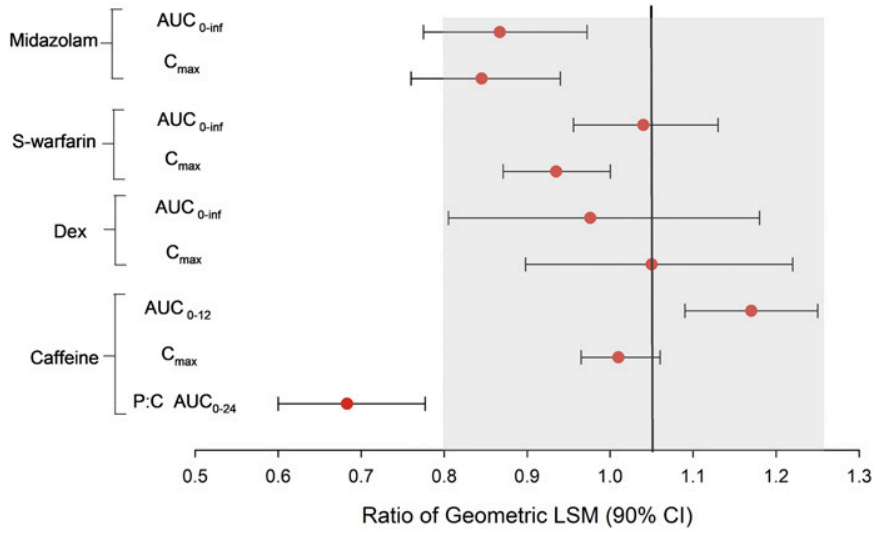

Fig. 5. Forest plot showing the change in $\mathrm{AUC}_{0-\text { inf }}$ and $\mathrm{C}_{\max }$ of midazolam, $S$-warfarin, and dextromethorphan; the $\mathrm{AUC}_{0-12}$ and $\mathrm{C}_{\max }$ of caffeine; and the paraxanthine:caffeine $\mathrm{AUC}_{0-24}$ ratio when taken with or without abemaciclib (200 mg Q12H for 7 days) after a CYP substrate drug cocktail containing $0.2 \mathrm{mg}$ midazolam (CYP3A4), $10 \mathrm{mg}$ warfarin (CYP2C19), $30 \mathrm{mg}$ dextromethorphan (CYP2D6), and $100 \mathrm{mg}$ caffeine (CYP1A2). $\mathrm{AUC}_{0-12}$, area under the concentration versus time curve from 0 to 12 hours; Dex, dextromethorphan; LSM, least squares mean.

(Wolenski et al., 2018). Despite the numerous examples of CYP downregulation in vitro, there is no clear example of CYP in vitro downregulation translating into meaningful DDIs in the clinic. The possible exceptions are 1) the effect of obeticholic acid on caffeine but not its metabolite paraxanthine (Edwards et al., 2017) and 2) the simultaneous inhibition and downregulation of CYP2D6 mediated by bupropion (Sager et al., 2017). Notably, the European Medicines Agency has reviewed a clinical package for a drug that exhibited downregulation of CYP activity both in vitro and in vivo (Hariparsad et al., 2017); however, no details of this package are publicly available at this time. Until sufficient knowledge and methodologies are developed to reliably translate in vitro CYP downregulation into clinical DDI predictions, any potentially concerning in vitro observations should be investigated further in the clinic.
For abemaciclib, which is extensively cleared by CYP3A4, clinical monotherapy PK data did not indicate any evidence of autoinhibition (http://uspl.lilly.com/verzenio/verzenio.html\#pi), suggesting abemaciclib would also not increase the exposure of the CYP3A4 substrate, midazolam. However, using the mechanistic static model with the in vitro downregulation data reported herein, a 2-fold change in midazolam AUC was predicted, driven predominantly by inhibition at the gut wall. Despite these contrasting findings and general absence in vitro-in vivo connectivity for CYP3A inhibition due to downregulation, the clinical investigation included midazolam to evaluate the effect of abemaciclib on the PK of CYP3A substrates. Indeed, the clinical study ruled out abemaciclib-mediated CYP3A inhibition, with marginal decreases in midazolam AUC, suggesting either an inconsequential degree of abemaciclib-mediated CYP3A4 induction or reduced absorption. Similarly, Wang and colleagues report in vitro downregulation of CYP3A4 mRNA by carfilzomib in human hepatocytes in vitro, which did not affect midazolam pharmacokinetics in patients with cancer $\left(\mathrm{AUC}_{0-\infty}\right.$ ratio [least squares mean $(90 \%$ geometric $\mathrm{CI})$ 108.2 (94.1-124)]) (Wang et al., 2013). Conversely, it was shown that weak induction of CYP3A-mediated midazolam clearance by the retinoid compounds was indeed predictable based on in vitro studies (Stevison et al., 2019). These studies again highlight the currently unreliable prediction of in vivo DDIs from in vitro data for CYP induction and/or downregulation. Notably, static models may overpredict drug interactions in cases of reversible inhibition and rapidly changing concentrations. However, a static model was deemed appropriate for this evaluation because 1) the downregulation is mainly occurring at the gut, where concentrations are high for a relatively short period generally coinciding with the absorption of midazolam;2) at steady state, abemaciclib and metabolites have relatively low fluctuation between $\mathrm{C}_{\max }$ and $\mathrm{C}_{\min }$, thus concentrations are not rapidly changing; and 3) changes due to downregulation would be expected to occur relatively slowly. That is, the long-enzyme turnover half-lives dampen the effect of changes in enzyme regulation.

For CYP2D6, the pathway is minimally regulated by transcription (Sager et al., 2017), and, consequently, the potential for induction is

TABLE 2

PK parameter estimates of CYP substrates

\begin{tabular}{|c|c|c|c|c|c|}
\hline \multirow[b]{2}{*}{ Midazolam (0.2 mg) } & \multicolumn{4}{|c|}{ Geometric Mean (CV\%) } & \multirow{2}{*}{ Ratio of Geometric LS Means (90\% CI) Test:reference } \\
\hline & $\mathrm{N}$ & Midazolam & $\mathrm{N}$ & Midazolam + abemaciclib & \\
\hline $\mathrm{AUC}_{0-\text { inf }}, \mathrm{ng} * \mathrm{~h} / \mathrm{ml}$ & 44 & $7.34(74)$ & 37 & $6.03(63)$ & $0.867(0.775,0.972)$ \\
\hline $\mathrm{C}_{\max }, \mathrm{ng} / \mathrm{ml}$ & 44 & $2.12(54)$ & 37 & $1.75(48)$ & $0.845(0.760,0.940)$ \\
\hline $\mathrm{t}_{\max }, \mathrm{h}^{a}$ & 44 & $0.50(0.40-1.07)$ & 37 & $0.50(0.33-2.17)$ & $0(0,0.0167)^{b}$ \\
\hline $\mathrm{t}_{1 / 2}, \mathrm{~h}$ & 44 & $7.62(2.23-31.20)$ & 37 & $6.33(3.27-13.00)$ & - \\
\hline S-warfarin $(10 \mathrm{mg})$ & & Warfarin & & Warfarin + abemaciclib & \\
\hline $\mathrm{AUC}_{0-\mathrm{inf}}, \mathrm{ng} * \mathrm{~h} / \mathrm{ml}$ & 44 & $21,400(43)$ & 30 & $20,600(40)$ & $1.04(0.956,1.13)$ \\
\hline $\mathrm{C}_{\max }, \mathrm{ng} / \mathrm{ml}$ & 44 & $561(35)$ & 30 & $526(35)$ & $0.935(0.871,1.00)$ \\
\hline $\mathrm{t}_{\max }, \mathrm{h}^{a}$ & 44 & $1.01(0.92-8.00)$ & 30 & $1.02(0.88-10.00)$ & $0.00835(0,0.0666)^{b}$ \\
\hline $\mathrm{t}_{1 / 2}, \mathrm{~h}$ & 44 & $43.0(25.70-132.00)$ & 30 & $42.2(25.80-81.80)$ & - \\
\hline Dextromethorphan $(30 \mathrm{mg})$ & & DEX & & $\mathrm{DEX}+$ abemaciclib & \\
\hline $\mathrm{AUC}_{0-\text { inf }}, \mathrm{ng} * \mathrm{~h} / \mathrm{ml}$ & 42 & $32.6(316)$ & 35 & $32.1(235)$ & $0.976(0.805,1.18)$ \\
\hline $\mathrm{C}_{\max }, \mathrm{ng} / \mathrm{ml}$ & 42 & $3.18(182)$ & 36 & $3.30(164)$ & $1.05(0.898,1.22)$ \\
\hline $\mathrm{t}_{\max }, \mathrm{h}^{a}$ & 42 & $2.08(0.93-6.00)$ & 36 & $2.03(1.07-10.25)$ & $0(0,0.0500)^{b}$ \\
\hline $\mathrm{t}_{1 / 2}, \mathrm{~h}$ & 42 & $10.90(4.48-26.00)$ & 35 & $9.44(4.83-17.00)$ & - \\
\hline Caffeine $(100 \mathrm{mg})$ & & Caffeine & & Caffeine + abemaciclib & \\
\hline $\mathrm{AUC}_{0-\mathrm{inf}}, \mathrm{ng} * \mathrm{~h} / \mathrm{ml}$ & 37 & $32,500(72)$ & 30 & $47,100(89)$ & $1.56(1.35,1.81)$ \\
\hline $\mathrm{C}_{\max }, \mathrm{ng} / \mathrm{ml}$ & 39 & $2890(29)$ & 32 & $2950(33)$ & $1.01(0.965,1.06)$ \\
\hline $\mathrm{t}_{\max }, \mathrm{h}^{a}$ & 39 & $0.52(0.40-3.00)$ & 32 & $0.53(0.37-22.30)$ & $0(-0.0500,0.250)$ \\
\hline $\mathrm{t}_{1 / 2}, \mathrm{~h}$ & 37 & $10.3(2.96-35.50)$ & 30 & $14.2(4.43-51.20)$ & - \\
\hline $\mathrm{P}: \mathrm{C} \mathrm{AUC}_{0-24}$ & 30 & $0.452(62)$ & 26 & $0.345(67)$ & $0.683(0.600,0.777)$ \\
\hline $\mathrm{P}: \mathrm{C} 6 \mathrm{~h}$ ratio & 30 & $0.404(76)$ & 26 & $0.287(79)$ & $0.611(0.516,0.722)$ \\
\hline
\end{tabular}

DEX, dextromethorphan; $t_{1 / 2}$, half-life.

$a_{\mathrm{t}_{\max }}$ is presented as median (min, max).

${ }^{b}$ Ratio of $\mathrm{t}_{\max }$ is presented as median of differences $(90 \% \mathrm{CI})$. 
rarely studied (Hariparsad et al., 2017). We thus expected no change in CYP2D6 mRNA levels after incubation with abemaciclib and metabolites. However, the in vitro data suggested mixed effects of abemaciclib on CYP2D6 induction at concentrations up to $5 \mu \mathrm{M}$ and downregulation at higher concentrations. Because there was no effect of multiple doses of $200 \mathrm{mg}$ abemaciclib Q12H on dextromethorphan [AUCR 0.976 $(0.805,1.18)]$, both the in vitro induction and downregulation appear to be false positives. The ability to predict in vivo CYP2D6 induction from in vitro studies is mixed: Sager et al. (2017) successfully predicted CYP2D6-mediated DDIs for bupropion based on simultaneous CYP2D6 inhibition and induction, but in vitro CYP2D6 downregulation by retinoids identified by Stevison et al. (2019) did not translate into the clinic.

The CYP1A2 substrate caffeine exhibited statistically significant increases in AUC, but the analysis was confounded by evidence of noncompliance with the dietary restriction of caffeine. For this reason, the caffeine metabolite, paraxanthine, was subsequently analyzed to determine the mechanism of any change in caffeine PK and to lessen the confounding effect of dietary caffeine consumption. The ratio of paraxanthine to caffeine (AUC or 6-hour concentrations) reflects the formation clearance of paraxanthine, the major CYP1A2 metabolite of caffeine, and has been used to quantify CYP1A2 activity (Tian et al., 2019). Using this approach, the present study suggests abemaciclib inhibits the CYP1A2-mediated metabolism of caffeine to paraxanthine. However, given the magnitude of intrasubject variability for caffeine AUC (34\%) (unpublished data), the inhibitory effect of abemaciclib on CYP1A2 is not considered clinically meaningful. This study highlights the inherent challenges in using caffeine as a substrate, especially in the outpatient setting wherein caffeine consumption cannot be controlled. Therefore, including the paraxanthine:caffeine ratio prospectively as a primary endpoint would be advised when caffeine is used as a probe substrate.

In conclusion, the downregulation of CYP mRNA expression and activity by abemaciclib in vitro did not translate into the clinic. No clinically relevant changes in the PK of CYP1A2, CYP2C9, CYP2D6, or CYP3A substrate drugs were observed when coadministered with multiple doses of abemaciclib. This lack of translation suggests the specific CYP mechanisms of downregulation in vitro need to be better understood, and further work is warranted to develop appropriate models to better inform clinical DDI risk.

\section{Acknowledgments}

The authors wish to acknowledge Jill Chappell for her expertise in study design and implementation.

\section{Authorship Contributions}

Participated in research design: Turner, Hall, Rehmel, Royalty, Guo, Kulanthaivel.

Conducted experiments: Rehmel.

Performed data analysis: Chapman, Rehmel.

Wrote or contributed to the writing of the manuscript: Turner, Hall, Chapman, Rehmel, Royalty, Guo, Kulanthaivel.

\section{References}

Birdwell KA, Decker B, Barbarino JM, Peterson JF, Stein CM, Sadee W, Wang D, Vinks AA, He Y, Swen JJ, et al. (2015) Clinical pharmacogenetics implementation consortium (CPIC) guidelines for CYP3A5 genotype and tacrolimus dosing. Clin Pharmacol Ther 98:19-24.

Burk O, Kuzikov M, Kronenberger T, Jeske J, Keminer O, Thasler WE, Schwab M, Wrenger C, and Windshügel B (2018) Identification of approved drugs as potent inhibitors of pregnane X receptor activation with differential receptor interaction profiles. Arch Toxicol 92:1435-1451.

Chainuvati S, Nafziger AN, Leeder JS, Gaedigk A, Kearns GL, Sellers E, Zhang Y, Kashuba AD, Rowland E, and Bertino JS Jr. (2003) Combined phenotypic assessment of cytochrome p450 $1 \mathrm{~A} 2,2 \mathrm{C} 9,2 \mathrm{C} 19,2 \mathrm{D} 6$, and $3 \mathrm{~A}, \mathrm{~N}$-acetyltransferase-2, and xanthine oxidase activities with the "Cooperstown 5+1 cocktail". Clin Pharmacol Ther 74:437-447.
Dickler MN, Tolaney SM, Rugo HS, Cortés J, Diéras V, Patt D, Wildiers H, Hudis CA, O'Shaughnessy J, Zamora E, et al. (2017) MONARCH 1, a phase II study of abemaciclib, a CDK4 and CDK6 inhibitor, as a single agent, in patients with refractory HR+/HER2- metastatic breast cancer. Clin Cancer Res 23:5218-5224.

Edwards JE, Eliot L, Parkinson A, Karan S, and MacConell L (2017) Assessment of pharmacokinetic interactions between obeticholic acid and caffeine, midazolam, warfarin, dextromethorphan, omeprazole, rosuvastatin, and digoxin in phase 1 studies in healthy subjects. $A d v$ Ther 34:2120-2138.

Fahmi OA, Maurer TS, Kish M, Cardenas E, Boldt S, and Nettleton D (2008) A combined model for predicting CYP3A4 clinical net drug-drug interaction based on CYP3A4 inhibition, inactivation, and induction determined in vitro. Drug Metab Dispos 36:1698-1708.

Fahmi OA and Ripp SL (2010) Evaluation of models for predicting drug-drug interactions due to induction. Expert Opin Drug Metab Toxicol 6:1399-1416.

Fijal BA, Guo Y, Li SG, Ahl J, Goto T, Tanaka Y, Nisenbaum LK, and Upadhyaya HP (2015) CYP2D6 predicted metabolizer status and safety in adult patients with attention-deficit hyperactivity disorder participating in a large placebo-controlled atomoxetine maintenance of response clinical trial. J Clin Pharmacol 55:1167-1174.

Hariparsad N, Ramsden D, Palamanda J, Dekeyser JG, Fahmi OA, Kenny JR, Einolf H, Mohutsky M, Pardon M, Siu YA, et al. (2017) Considerations from the IQ induction working group in response to drug-drug interaction guidance from regulatory agencies: focus on downregulation, CYP2C induction, and CYP2B6 positive control. Drug Metab Dispos 45:1049-1059 DOI: 10.1124/dmd.116.074567.

Hicks JK, Sangkuhl K, Swen JJ, Ellingrod VL, Müller DJ, Shimoda K, Bishop JR, Kharasch ED, Skaar TC, Gaedigk A, et al. (2017) Clinical pharmacogenetics implementation consortium guideline (CPIC) for CYP2D6 and CYP2C19 genotypes and dosing of tricyclic antidepressants: 2016 update. Clin Pharmacol Ther 102:37-44.

Ingelman-Sundberg M, Sim SC, Gomez A, and Rodriguez-Antona C (2007) Influence of cytochrome $\mathrm{P} 450$ polymorphisms on drug therapies: pharmacogenetic, pharmacoepigenetic and clinical aspects. Pharmacol Ther 116:496-526.

Ito K, Iwatsubo T, Kanamitsu S, Ueda K, Suzuki H, and Sugiyama Y (1998) Prediction of pharmacokinetic alterations caused by drug-drug interactions: metabolic interaction in the liver. Pharmacol Rev 50:387-412.

Johnson JA, Gong L, Whirl-Carrillo M, Gage BF, Scott SA, Stein CM, Anderson JL, Kimmel SE, Lee MT, Pirmohamed M, et al.; Clinical Pharmacogenetics Implementation Consortium (2011) Clinical pharmacogenetics implementation consortium guidelines for CYP2C9 and VKORC1 genotypes and warfarin dosing. Clin Pharmacol Ther 90:625-629.

Kulanthaivel P, Mahadevan D, Turner PK, Royalty J, Ng WT, Yi P, Rehmel J, Cassidy K, and Chappell J (2016) Pharmacokinetic drug interactions between abemaciclib and CYP3A inducers and inhibitors, in Proceedings of the 107th Annual Meeting of the American Association of Cancer Research; 2016 April 16-20; New Orleans, LA. Vol 13, pp A586.

Patnaik A, Rosen LS, Tolaney SM, Tolcher AW, Goldman JW, Gandhi L, Papadopoulos KP, Beeram M, Rasco DW, Hilton JF, et al. (2016) Efficacy and safety of abemaciclib, an inhibitor of CDK4 and CDK6, for patients with breast cancer, non-small cell lung cancer, and other solid tumours. Cancer Discov 6:740-753.

Posada MM, Turner PK, Kulanthaivel P, Hall SD, and Dickinson GL (2017) Predicting clinical effects of CYP3A perpetrators on abemaciclib and active metabolites exposure using physiologically-based pharmacokinetic modelling. in American Society for Clinical Pharmacology and Therapeutics Annual Meeting; 2017 March 15-18.

Rostami-Hodjegan A and Tucker G (2004) 'In silico' simulations to assess the 'in vivo' consequences of 'in vitro' metabolic drug-drug interactions. Drug Discov Today Technol 1:441-448. Sager JE, Tripathy S, Price LSL, Nath A, Chang J, Stephenson-Famy A, and Isoherranen N (2017) In vitro to in vivo extrapolation of the complex drug-drug interaction of bupropion and its metabolites with CYP2D6; simultaneous reversible inhibition and CYP2D6 downregulation. Biochem Pharmacol 123:85-96.

Staudinger JL (2019) Clinical applications of small molecule inhibitors of Pregnane X receptor. Mol Cell Endocrinol 485:61-71.

Stevison F, Kosaka M, Kenny JR, Wong S, Hogarth C, Amory JK, and Isoherranen N (2019) Does in vitro cytochrome $\mathrm{P} 450$ downregulation translate to in vivo drug-drug interactions? Preclinical and clinical studies with 13-cis-retinoic acid. Clin Transl Sci 12:350-360 DOI: 10.1111/ cts. 12616.

Sun Y, Chothe PP, Sager JE, Tsao H, Moore A, Laitinen L, and Hariparsad N (2017) Quantitative prediction of CYP3A4 induction: impact of measured, free, and intracellular perpetrator concentrations from human hepatocyte induction studies on drug-drug interaction predictions. Drug Metab Dispos 45:692-705.

Tate SC, Sykes AK, Kulanthaivel P, Chan EM, Turner PK, and Cronier DM (2018) A population pharmacokinetic and pharmacodynamic analysis of abemaciclib in a phase 1 clinical trial in cancer patients. Clin Pharmacokinet 57:335-344.

Tian DD, Natesan S, White JR Jr., and Paine MF (2019) Effects of common CYP1A2 genotypes and other key factors on intraindividual variation in the caffeine metabolic ratio: an exploratory analysis. Clin Transl Sci 12:39-46.

Tian Y (2013) Epigenetic regulation of pregnane X receptor activity. Drug Metab Rev 45:166-172. United States Food and Drug Administration (2017) Clinical drug interaction studies-Study design, data analysis, and clinical implications guidance for industry. Available at: https://www.fda.gov/ downloads/drugs/guidances/ucm292362.pdf. Accessed 22-Apr-2019.

Wang Z, Yang J, Kirk C, Fang Y, Alsina M, Badros A, Papadopoulos K, Wong A, Woo T, Bomba $\mathrm{D}$, et al. (2013) Clinical pharmacokinetics, metabolism, and drug-drug interaction of carfilzomib. Drug Metab Dispos 41:230-237.

Wolenski FS, Xia CQ, Ma B, Han TH, Shyu WC, and Balani SK (2018) CYP suppression in human hepatocytes by monomethyl auristatin $\mathrm{E}$, the payload in brentuximab vedotin (Adcetris $($ ), is associated with microtubule disruption. Eur J Drug Metab Pharmacokinet 43:347-354.

Yang J, Liao M, Shou M, Jamei M, Yeo KR, Tucker GT, and Rostami-Hodjegan A (2008) Cytochrome p450 turnover: regulation of synthesis and degradation, methods for determining rates, and implications for the prediction of drug interactions. Curr Drug Metab 9:384-394.

Address correspondence to: P. Kellie Turner, Eli Lilly and Company, Lilly Corporate Center, Indianapolis, IN 46285. E-mail: turnerpa@lilly.com 